\title{
On the Study of Transience and Recurrence of the Markov Chain Defined by Directed Weighted Circuits Associated with a Random Walk in Fixed Environment
}

\author{
Chrysoula Ganatsiou \\ Department of Civil Engineering, Section of Mathematics and Statistics, School of Technological Sciences, \\ University of Thessaly, Pedion Areos, 38334 Volos, Greece \\ Correspondence should be addressed to Chrysoula Ganatsiou; ganatsio@otenet.gr
}

Received 23 October 2012; Revised 2 March 2013; Accepted 27 March 2013

Academic Editor: Man Lai Tang

Copyright (C) 2013 Chrysoula Ganatsiou. This is an open access article distributed under the Creative Commons Attribution License, which permits unrestricted use, distribution, and reproduction in any medium, provided the original work is properly cited.

By using the cycle representation theory of Markov processes, we investigate proper criterions regarding transience and recurrence of the corresponding Markov chain represented uniquely by directed cycles (especially by directed circuits) and weights of a random walk with jumps in a fixed environment.

\section{Introduction}

A systematic research has been developed (Kalpazidou [1], MacQueen [2], Minping and Min [3], Zemanian [4], and others) in order to investigate representations of the finitedimensional distributions of Markov processes (with discrete or continuous parameter) having an invariant measure, as decompositions in terms of the cycle (or circuit) passage functions:

$$
\begin{aligned}
J_{\widehat{c}}(i, j) & =1, \quad \text { if } i, j \text { are consecutive states of } \widehat{c}, \\
& =0, \quad \text { otherwise, }
\end{aligned}
$$

for any directed sequence $\widehat{c}=\left(i_{1}, i_{2}, \ldots, i_{r}\right)$ (or $c=$ $\left.\left(i_{1}, i_{2}, \ldots, i_{r}, i_{1}\right)\right)$ of states called a cycle (or a circuit), $r>1$, of the corresponding Markov process. The representations are called cycle (or circuit) representations while the corresponding discrete parameter Markov processes generated by directed circuits $c=\left(i_{1}, i_{2}, \ldots, i_{r}, i_{1}\right), r>1$, are called circuit chains.

Following the context of the theory of Markov processes' cycle-circuit representation, the present work arises as an attempt to investigate proper criterions regarding the properties of transience and recurrence of the corresponding Markov chain represented uniquely by directed cycles (especially by directed circuits) and weights of a random walk with jumps (having one elastic left barrier) in a fixed ergodic environment (Kalpazidou [1], Derriennic [5]).

The paper is organized as follows. In Section 2, we give a brief account of certain concepts of cycle representation theory of Markov processes that we will need throughout the paper. In Section 3, we present some auxiliary results in order to make the presentation of the paper more comprehensible. In particular, in Section 3, a random walk with jumps (having one elastic left barrier) in a fixed ergodic environment is considered, and the unique representations by directed cycles (especially by directed circuits) and weights of the corresponding Markov chain are investigated. These representations will give us the possibility to study proper criterions regarding transience and recurrence of the abovementioned Markov chain, as it is described in Section 4.

Throughout the paper, we will need the following notations:

$$
\begin{gathered}
\aleph=\{0,1,2, \ldots\}, \quad \aleph^{*}=\{1,2, \ldots\}, \\
Z=\{\ldots,-1,0,1, \ldots\} .
\end{gathered}
$$

\section{Preliminaries}

Let us consider a denumerable set $\mathrm{S}$. Then the directed sequence $c=\left(i_{1}, i_{2}, \ldots, i_{r}, i_{1}\right)$ modulo the cyclic permutations, where $i_{1}, i_{2}, \ldots, i_{r} \in S, r>1$, completely defines a 
directed circuit in $S$. The ordered sequence $\widehat{c}=\left(i_{1}, i_{2}, \ldots, i_{r}\right)$ associated with the given directed $c$ is called a directed cycle in $S$.

A directed circuit may be considered as $c=(c(r), c(r+$ 1), .., $c(r+v-1), c(r+v))$, if there exists an $r \in Z$, such that $i_{1}=c(r+0), i_{2}=c(r+1), \ldots, i_{v}=c(r+v-1), i_{1}=c(r+v)$, where $c$ is a periodic function from $Z$ to $S$.

The corresponding directed cycle is defined by the ordered sequence $\widehat{c}=(c(r), c(r+1), \ldots, c(r+v-1))$. The values $c(k)$ are the points of $c$, while the directed pairs $(c(k), c(k+1))$, $k \in Z$, are the directed edges of $c$.

The smallest integer $p \equiv p(c) \geq 1$ satisfying the equation $c(r+p)=c(r)$, for all $r \in Z$, is the period of $c$. A directed circuit $c$ such that $p(c)=1$ is called a loop. (In the present work we will use directed circuits with distinct point elements.)

Let us also consider a directed circuit $c$ (or a directed cycle $\widehat{c})$ with period $p(c)>1$. Then we may define by

$$
\begin{array}{r}
J_{c}^{(n)}(i, j)=1, \quad \text { if there exists an } r \in Z \text { such that } \\
i=c(r), j=c(r+n),
\end{array}
$$

$=0$, otherwise,

the $n$-step passage function associated with the directed circuit $c$, for any $i, j \in S, n \geq 1$.

Furthermore we may define by

$$
\begin{aligned}
J_{c}(i) & =1, \quad \text { if there exists an } r \in Z \text { such that } i=c(r), \\
& =0, \quad \text { otherwise, }
\end{aligned}
$$

the passage function associated with the directed circuit $c$, for any $i \in S$. The above definitions are due to MacQueen [2] and Kalpazidou [1].

Given a denumerable set $S$ and an infinite denumerable class $C$ of overlapping directed circuits (or directed cycles) with distinct points (except for the terminals) in $S$ such that all the points of $S$ can be reached from one another following paths of circuit edges; that is, for each two distinct points $i$ and $j$ of $S$ there exists a finite sequence $c_{1}, \ldots, c_{k}, k \geq 1$, of circuits (or cycles) of $C$ such that $i$ lies on $c_{1}$ and $j$ lies on $c_{k}$, and any pair of consecutive circuits $\left(c_{n}, c_{n+1}\right)$ have at least one point in common. Generally we may assume that the class $C$ contains, among its elements, circuits (or cycles) with period greater or equal to 2 .

With each directed circuit (or directed cycle) $c \in C$ let us associate a strictly positive weight $w_{c}$ which must be independent of the choice of the representative of $c$, that is, it must satisfy the consistency condition $w_{\text {cot }_{k}}=w_{c}, k \in Z$, where $t_{k}$ is the translation of length $k$ (that is, $t_{k}(n) \equiv n+k$, $n \in Z$, for any fixed $k \in Z$ ).

For a given class $C$ of overlapping directed circuits (or cycles) and for a given sequence $\left(w_{c}\right)_{c \in C}$ of weights we may define by

$$
p_{i j}=\frac{\sum_{c \in C} w_{c} \cdot J_{c}^{(1)}(i, j)}{\sum_{c \in C} w_{c} \cdot J_{c}(i)}
$$

the elements of a Markov transition matrix on $S$, if and only if $\sum_{c \in C} w_{c} \cdot J_{c}(i)<\infty$, for any $i \in S$. This means that a given Markov transition matrix $P=\left(p_{i j}\right), i, j \in S$, can be represented by directed circuits (or cycles) and weights if and only if there exists a class of overlapping directed circuits (or cycles) $C$ and a sequence of positive weights $\left(w_{c}\right)_{c \in C}$ such that the formula (5) holds. In this case, the Markov transition matrix $P$ has a unique stationary distribution $p$ which is a solution of $p P=p$ and is defined by

$$
p(i)=\sum_{c \in C} w_{c} \cdot J_{c}(i), \quad i \in S
$$

It is known that the following classes of Markov chains may be represented uniquely by circuits (or cycles) and weights:

(i) the recurrent Markov chains (Minping and Min [3]),

(ii) the reversible Markov chains.

\section{Auxiliary Results}

Let us consider a Markov chain $\left(X_{n}\right)_{n \geq 0}$ on $\aleph$ with transitions $k \rightarrow(k+1), k \rightarrow(k-1)$, and $k \rightarrow k$ whose the elements of the corresponding Markov transition matrix are defined by

$$
\begin{gathered}
P\left(X_{n+1}=0 / X_{n}=0\right)=r_{0}, \\
P\left(X_{n+1}=1 / X_{n}=0\right)=p_{0}, \quad p_{0}=1-r_{0}, \\
P\left(X_{n+1}=k+1 / X_{n}=k\right)=p_{k}, \quad k \geq 1, \\
P\left(X_{n+1}=k / X_{n}=k\right)=r_{k}, \quad k \geq 1, \\
P\left(X_{n+1}=k-1 / X_{n}=k\right)=q_{k}, \quad k \geq 1,
\end{gathered}
$$

such that $p_{k}+q_{k}+r_{k}=1,0 \leq p_{k}, r_{k} \leq 1$, for every $k \geq 1$, as it is shown in (Figure 1).

Assume that $\left(p_{k}\right)_{k \geq 0}$ and $\left(r_{k}\right)_{k \geq 0}$ are arbitrary fixed sequences with $0 \leq p_{0}=1-r_{0} \leq 1,0 \leq p_{k}, r_{k} \leq 1$, for every $k>1$. If we consider the directed circuits $c_{k}=(k, k+1, k)$, $c_{k}^{\prime}=(k, k), k \geq 0$, and the collections of weights $\left(w_{c_{k}}\right)_{k \geq 0}$ and $\left(w_{c_{k}^{\prime}}\right)_{k \geq 0}$, respectively, then we may obtain that

$$
p_{k}=\frac{w_{c_{k}}}{w_{c_{k-1}}+w_{c_{k}}+w_{c_{k}^{\prime}}}, \quad \text { for every } k \geq 1,
$$

with

$$
p_{0}=\frac{w_{c_{0}}}{w_{c_{0}}+w_{c_{0}^{\prime}}} .
$$

Here the class $C(k)$ contains the directed circuits $c_{k}=$ $(k, k+1, k), c_{k-1}=(k-1, k, k-1)$, and $c_{k}^{\prime}=(k, k)$. Furthermore we may define

$$
q_{k}=\frac{w_{c_{k-1}}}{w_{c_{k-1}}+w_{c_{k}}+w_{c_{k}^{\prime}}}, \quad \text { for every } k \geq 1
$$

and $r_{k}=w_{c_{k}^{\prime}} /\left(w_{c_{k-1}}+w_{c_{k}}+w_{c_{k}^{\prime}}\right)$, such that $p_{k}+q_{k}+r_{k}=1$, for every $k \geq 1$, with $r_{0}=1-p_{0}=w_{c_{0}^{\prime}} /\left(w_{c_{0}}+w_{c_{0}^{\prime}}\right)$. 


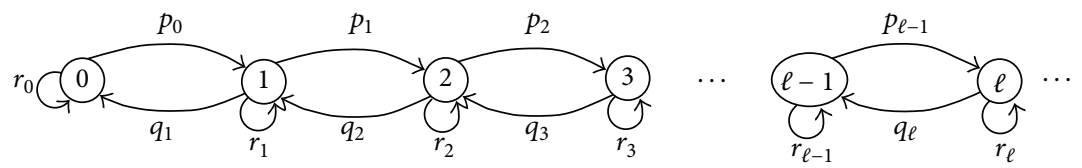

FigURE 1

The transition matrix $P=\left(p_{i j}\right)$ with

$$
\begin{gathered}
p_{i j}=\frac{\sum_{k=0}^{\infty} w_{\mathcal{c}_{k}} \cdot J_{c_{k}}^{(1)}(i, j)}{\sum_{k=0}^{\infty}\left[w_{\mathcal{c}_{k}} \cdot J_{\mathcal{c}_{k}}(i)+w_{c_{k}^{\prime}} \cdot J_{\mathcal{c}_{k}^{\prime}}(i)\right]}, \quad \text { for } i \neq j, \\
p_{i i}=\frac{\sum_{k=0}^{\infty} w_{c_{k}} \cdot J_{c_{k}^{\prime}}^{(1)}(i, i)}{\sum_{k=0}^{\infty}\left[w_{c_{k}} \cdot J_{\mathcal{c}_{k}}(i)+w_{\mathcal{c}_{k}^{\prime}} \cdot J_{c_{k}^{\prime}}(i)\right]},
\end{gathered}
$$

where $J_{\mathcal{c}_{k}}^{(1)}(i, j)=1$, if $i, j$ are consecutive points of the circuit $c_{k}, J_{c_{k}}(i)=1$, if $i$ is a point of the circuit $c_{k}$, and $J_{c_{k}^{\prime}}(i)=1$, if $i$ is a point of the circuit $c_{k}^{\prime}$, expresses the representation of the Markov chain $\left(X_{n}\right)_{n \geq 0}$ by directed cycles (especially by directed circuits) and weights.

Furthermore we consider also the "adjoint" Markov chain $\left(X_{n}^{\prime}\right)_{n \geq 0}$ on $\aleph$ whose elements of the corresponding Markov transition matrix are defined by

$$
\begin{aligned}
& P\left(X_{n+1}^{\prime}=0 / X_{n}^{\prime}=0\right)=r_{0}^{\prime}, \\
& P\left(X_{n+1}^{\prime}=1 / X_{n}^{\prime}=0\right)=q_{0}^{\prime}, \quad q_{0}^{\prime}=1-r_{0}^{\prime}, \\
& P\left(X_{n+1}^{\prime}=k-1 / X_{n}^{\prime}=k\right)=p_{k}^{\prime}, \quad k \geq 1, \\
& P\left(X_{n+1}^{\prime}=k / X_{n}^{\prime}=k\right)=r_{k}^{\prime}, \quad k \geq 1, \\
& P\left(X_{n+1}^{\prime}=k+1 / X_{n}^{\prime}=k\right)=q_{k}^{\prime}, \quad k \geq 1,
\end{aligned}
$$

such that $p_{k}^{\prime}+q_{k}^{\prime}+r_{k}^{\prime}=1,0<p_{k}^{\prime}, q_{k}^{\prime}, r_{k}^{\prime} \leq 1$, for every $k \geq 1$, as it is shown in (Figure 2).

Assume that $\left(q_{k}^{\prime}\right)_{k \geq 0}$ and $\left(r_{k}^{\prime}\right)_{k \geq 0}$ are arbitrary fixed sequences with $0 \leq q_{0}^{\prime}=1-r_{0}^{\prime} \leq 1,0 \leq q_{k}^{\prime}, r_{k}^{\prime} \leq 1$, for every $k \geq 1$. If we consider the directed circuits $c_{k}^{\prime \prime}=(k+1, k, k+1)$, $c_{k}^{\prime \prime \prime}=(k, k), k \geq 0$, and the collections of weights $\left(w_{c_{k}^{\prime \prime}}\right)_{k \geq 0}$, and $\left(w_{c_{k}^{\prime \prime \prime}}\right)_{k \geq 0}$ respectively, then we may have

$$
q_{k}^{\prime}=\frac{w_{c_{k}^{\prime \prime}}}{w_{c_{k-1}^{\prime \prime}}+w_{c_{k}^{\prime \prime}}+w_{c_{k}^{\prime \prime \prime}}}, \quad \text { for every } k \geq 1 \text {, }
$$

with

$$
q_{0}^{\prime}=\frac{w_{c_{0}^{\prime \prime}}}{w_{c_{0}^{\prime \prime}}+w_{c_{0}^{\prime \prime \prime}}} .
$$

Here the class $C^{\prime}(k)$ contains the directed circuits $c_{k}^{\prime \prime}=$ $(k+1, k, k+1), c_{k-1}^{\prime \prime}=(k, k-1, k)$, and $c_{k}^{\prime \prime \prime}=(k, k)$. Furthermore we may define

$$
\begin{gathered}
p_{k}^{\prime}=\frac{w_{c_{k-1}^{\prime \prime}}}{w_{c_{k-1}}^{\prime \prime}+w_{c_{k}^{\prime \prime}}+w_{c_{k}^{\prime \prime \prime}}}, \quad \text { for every } k \geq 1, \\
r_{k}^{\prime}=\frac{w_{c_{k}^{\prime \prime \prime}}}{w_{c_{k-1}^{\prime \prime}}+w_{c_{k}^{\prime \prime}}+w_{c_{k}^{\prime \prime \prime}}},
\end{gathered}
$$

such that $p_{k}^{\prime}+q_{k}^{\prime}+r_{k}^{\prime}=1$, for every $k \geq 1$, with

$$
r_{0}^{\prime}=1-q_{0}^{\prime}=\frac{w_{c_{0}^{\prime \prime \prime}}}{w_{c_{0}^{\prime \prime}}+w_{c_{0}^{\prime \prime \prime}}} .
$$

So the transition matrix $P^{\prime}=\left(p_{i j}^{\prime}\right)$ with elements equivalent to that given by the above-mentioned formulas (11), (12) expresses also the representation of the "adjoint" Markov chain $\left(X_{n}^{\prime}\right)_{n>0}$ by directed cycles (especially by directed circuits) and weights.

Consequently we have the following.

Proposition 1. The Markov chain $\left(X_{n}\right)_{n \geq 0}$ defined as above has a unique representation by directed cycles (especially by directed circuits) and weights.

Proof. Let us consider the set of directed circuits $c_{k}=(k, k+$ $1, k)$ and $c_{k}^{\prime}=(k, k)$, for every $k \geq 0$, since only the transitions from $k$ to $k+1, k$ to $k-1$, and $k$ to $k$ are possible. There are three circuits through each point $k \geq 1, c_{k-1}, c_{k}$, and $c_{k}^{\prime}$ and two circuits through $0: c_{0}, c_{0}^{\prime}$.

The problem we have to manage is the definition of the weights. We may symbolize by $w_{k}$ the weight $w_{c_{k}}$ of the circuit $c_{k}$ and by $w_{k}^{\prime}$ the weight $w_{c_{k}^{\prime}}$ of the circuit $c_{k}^{\prime}$, for any $k \geq 0$. The sequences $\left(w_{k}\right)_{k \geq 0},\left(w_{k}^{\prime}\right)_{k \geq 0}$ must be a solution of

$$
\begin{gathered}
p_{k}=\frac{w_{k}}{w_{k-1}+w_{k}+w_{k}^{\prime}}, \quad k \geq 1 \text { with } p_{0}=\frac{w_{0}}{w_{0}+w_{0}^{\prime}}, \\
r_{k}=\frac{w_{k}^{\prime}}{w_{k-1}+w_{k}+w_{k}^{\prime}}, \quad k \geq 1 \text { with } r_{0}=\frac{w_{0}^{\prime}}{w_{0}+w_{0}^{\prime}}, \\
q_{k}=1-p_{k}-r_{k}, \quad k \geq 1 .
\end{gathered}
$$

Let us take by $b_{k}=w_{k} / w_{k-1}, \gamma_{k}=w_{k}^{\prime} / w_{k-1}^{\prime}, k \geq 1$. As a consequence we may have

$$
b_{k}=\frac{p_{k}}{q_{k}}=\frac{p_{k}}{1-p_{k}-r_{k}},
$$$$
\gamma_{k}=\frac{r_{k}}{r_{k-1}} \frac{p_{k-1}}{p_{k}} b_{k}, \quad \text { for every } k \geq 1 \text {. }
$$ 


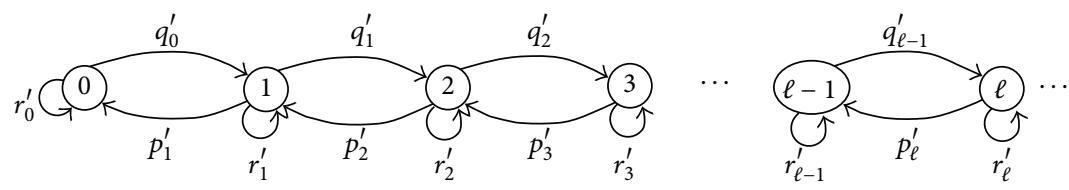

FiguRe 2

Given the sequences $\left(p_{k}\right)_{k \geq 0}$ and $\left(r_{k}\right)_{k \geq 0}$, it is clear that the above sequences $\left(b_{k}\right)_{k \geq 1}$ and $\left(\gamma_{k}\right)_{k \geq 1}$ exist and are unique. This means that the sequences $\left(w_{k}\right)_{k \geq 0}$ and $\left(w_{k}^{\prime}\right)_{k \geq 0}$ are defined uniquely, up to multiplicative constant factors, by

$$
\begin{aligned}
& w_{k}=w_{0} \cdot b_{1} \cdots b_{k}, \\
& w_{k}^{\prime}=w_{0}^{\prime} \cdot \gamma_{1} \cdots \gamma_{k} .
\end{aligned}
$$
$w_{0}^{\prime}$.)

(The unicity is understood up to the constant factors $w_{0}$,

Proposition 2. The "adjoint" Markov chain $\left(X_{n}^{\prime}\right)_{n \geq 0}$ defined as above has a unique representation by directed cycles (especially by directed circuits) and weights.

Proof. Following an analogous way of that given in the proof of Proposition 1 the problem we have also to manage here is the definition of the weights. To this direction we may symbolize by $w_{k}^{\prime \prime}$ the weight $w_{c_{k}}^{\prime \prime}$ of the circuit $c_{k}^{\prime \prime}$ and by $w_{k}^{\prime \prime \prime}$ the weight $w_{c_{k}}^{\prime \prime \prime}$ of the circuit $c_{k}^{\prime \prime \prime}$, for every $k \geq 0$. The sequences $\left(w_{k}^{\prime \prime}\right)_{k \geq 0}$ and $\left(w_{k}^{\prime \prime \prime}\right)_{k \geq 0}$ must be solutions of

$$
\begin{gathered}
q_{k}^{\prime}=\frac{w_{k}^{\prime \prime}}{w_{k-1}^{\prime \prime}+w_{k}^{\prime \prime}+w_{k}^{\prime \prime \prime}}, \quad k \geq 1 \text { with } q_{0}^{\prime}=\frac{w_{0}^{\prime \prime}}{w_{0}^{\prime \prime}+w_{0}^{\prime \prime \prime}}, \\
r_{k}^{\prime}=\frac{w_{k}^{\prime \prime \prime}}{w_{k-1}^{\prime \prime}+w_{k}^{\prime \prime}+w_{k}^{\prime \prime \prime}}, \quad k \geq 1 \text { with } r_{0}^{\prime}=\frac{w_{0}^{\prime \prime \prime}}{w_{0}^{\prime \prime}+w_{0}^{\prime \prime \prime}}, \\
p_{k}^{\prime}=1-q_{k}^{\prime}-r_{k}^{\prime}, \quad k \geq 1 .
\end{gathered}
$$

By considering the sequences $\left(s_{k}\right)_{k}$ and $\left(t_{k}\right)_{k}$ where $s_{k}=$ $w_{k-1}^{\prime \prime} / w_{k}^{\prime \prime}, t_{k}=w_{k}^{\prime \prime \prime} / w_{k-1}^{\prime \prime \prime}, k \geq 1$, we may obtain that

$$
\begin{gathered}
s_{k}=\frac{1-q_{k}^{\prime}-r_{k}^{\prime}}{q_{k}^{\prime}}, \\
t_{k}=\frac{r_{k}^{\prime}}{r_{k-1}^{\prime}} \cdot \frac{q_{k}^{\prime}}{q_{k-1}^{\prime}} \cdot s_{k}, \quad \text { for every } k \geq 1 .
\end{gathered}
$$

For given sequences $\left(q_{k}^{\prime}\right)_{k \geq 0},\left(r_{k}^{\prime}\right)_{k \geq 0}$ it is obvious that $\left(s_{k}\right)_{k \geq 1},\left(t_{k}\right)_{k \geq 1}$ exist and are unique for those sequences, that is, the sequences $\left(w_{k}^{\prime \prime}\right)_{k \geq 0},\left(w_{k}^{\prime \prime \prime}\right)_{k \geq 0}$ are defined uniquely, up to multiplicative constant factors, by

$$
\begin{gathered}
w_{k}^{\prime \prime}=\frac{w_{0}^{\prime \prime}}{s_{1} \cdot s_{2} \cdots s_{k}}, \\
w_{k}^{\prime \prime \prime}=w_{0}^{\prime \prime \prime} \cdot t_{1}, t_{2} \cdots t_{k} .
\end{gathered}
$$

(The unicity is based to the constant factors $w_{0}^{\prime \prime}, w_{0}^{\prime \prime \prime}$.)

\section{Recurrence and Transience of the Markov Chains $\left(X_{n}\right)_{n \geq 0}$ and $\left(X_{n}^{\prime}\right)_{n \geq 0}$}

We have that for the Markov chain $\left(X_{n}\right)_{n \geq 0}$, there is a unique invariant measure up to a multiplicative constant factor $\mu_{k}=$ $w_{k-1}+w_{k}+w_{k}^{\prime}, \quad k \geq 1, \mu_{0}=w_{0}+w_{0}^{\prime}$, while for the Markov chain $\left(X_{n}^{\prime}\right)_{n \geq 0}, \mu_{k}^{\prime}=w_{k-1}^{\prime \prime}+w_{k}^{\prime \prime}+w_{k}^{\prime \prime \prime}, k \geq 1$ with $\mu_{0}^{\prime}=w_{0}^{\prime \prime}+w_{0}^{\prime \prime \prime}$. In the case that an irreducible chain is recurrent there is only one invariant measure (finite or not), so we may obtain the following.

Proposition 3. (i) The Markov chain $\left(X_{n}\right)_{n \geq 0}$ defined as above is positive recurrent if and only if

$$
\begin{array}{ll}
\sum_{k=1}^{\infty} b_{1} \cdot b_{2} \cdots b_{k}<+\infty & \left(\text { or } \frac{1}{w_{0}} \cdot \sum_{k=1}^{\infty} w_{k}<+\infty\right), \\
\sum_{k=1}^{\infty} \gamma_{1} \cdot \gamma_{2} \cdots \gamma_{k}<+\infty & \left(\text { or } \frac{1}{w_{0}^{\prime}} \cdot \sum_{k=1}^{\infty} w_{k}^{\prime}<+\infty\right) .
\end{array}
$$

(ii) The Markov chain $\left(X_{n}^{\prime}\right)_{n \geq 0}$ defined as above is positive recurrent if and only if

$$
\begin{array}{ll}
\sum_{k=1}^{\infty} \frac{1}{s_{1} \cdot s_{2} \cdots s_{k}}<+\infty & \left(\text { or } \frac{1}{w_{0}^{\prime \prime}} \cdot \sum_{k=1}^{\infty} w_{k}^{\prime \prime}<+\infty\right), \\
\sum_{k=1}^{\infty} t_{1} \cdot t_{2} \cdots t_{k}=+\infty & \left(\text { or } \frac{1}{w_{0}^{\prime \prime \prime}} \cdot \sum_{k=1}^{\infty} w_{k}^{\prime \prime \prime}=+\infty\right) .
\end{array}
$$

In order to obtain recurrence and transience criterions for the Markov chains $\left(X_{n}\right)_{n \geq 0}$ and $\left(X_{n}^{\prime}\right)_{n \geq 0}$ we shall need the following proposition (Karlin and Taylor [6]).

Proposition 4. Let us consider a Markov chain on $\aleph$ which is irreducible. Then if there exists a strictly increasing function that is harmonic on the complement of a finite interval and that is bounded, then the chain is transient. In the case that there exists such a function which is unbounded then the chain is recurrent.

Following this direction, we shall use a well-known method-theorem based on the Foster-Kendall theorem (Karlin and Taylor [6]) by considering the harmonic function $g=\left(g_{k}, k \geq 1\right)$. For the Markov chain $\left(X_{n}\right)_{n \geq 0}$ this is a solution of

$$
\begin{gathered}
p_{0} \cdot g_{1}+r_{0} \cdot g_{0}=g_{0}, \\
p_{k} \cdot g_{k+1}+q_{k} \cdot g_{k-1}+r_{k} \cdot g_{k}=g_{k}, \quad k \geq 1 .
\end{gathered}
$$


Since $\Delta g_{k}=g_{k}-g_{k-1}$, for every $k \geq 1$, we obtain that

$$
\begin{gathered}
p_{k} \cdot g_{k+1}+q_{k} \cdot g_{k} \\
-q_{k} \cdot g_{k}+q_{k} \cdot g_{k-1}+r_{k} \cdot g_{k}=g_{k}, \\
p_{k} \cdot\left(\Delta g_{k+1}+g_{k}\right)+q_{k} \cdot g_{k} \\
-q_{k} \cdot g_{k}+q_{k} \cdot g_{k-1}+r_{k} \cdot g_{k}=g_{k} \\
p_{k} \cdot \Delta g_{k+1}+\left(p_{k}+q_{k}+r_{k}\right) \cdot g_{k} \\
-q_{k} \cdot g_{k}+q_{k} \cdot g_{k-1}=g_{k} \\
p_{k} \cdot \Delta g_{k+1}-q_{k} \cdot\left(g_{k}-g_{k-1}\right)=0 \\
p_{k} \cdot \Delta g_{k+1}=q_{k} \cdot \Delta g_{k} .
\end{gathered}
$$

If we put $m_{k}=\Delta g_{k} / \Delta g_{k+1}$, we get $m_{k}=p_{k} / q_{k}$ (with $\left.p_{k}=1-q_{k}-r_{k}\right), k \geq 1$, which is the equation of definition of the sequences $\left(s_{k}\right)_{k \geq 1}$ and $\left(t_{k}\right)_{k \geq 1}$ (as a multiplicative factor of the $\left.\left(s_{k}\right)_{k \geq 1}\right)$ for the Markov chain $\left(X_{n}^{\prime}\right)_{n \geq 0}$ such that $q_{k}^{\prime}=$ $q_{k}, r_{k}^{\prime}=r_{k}$, for every $k \geq 1$. This means that the strictly increasing harmonic functions of the Markov chain $\left(X_{n}\right)_{n \geq 0}$ are in correspondence with the weight representations of the Markov chain $\left(X_{n}^{\prime}\right)_{n \geq 0}$ such that

$$
\begin{aligned}
q_{k}^{\prime} & =P\left(X_{n+1}^{\prime}=k+1 / X_{n}^{\prime}=k\right) \\
& =P\left(X_{n+1}=k-1 / X_{n}=k\right)=q_{k}, \\
r_{k}^{\prime} & =P\left(X_{n+1}^{\prime}=k / X_{n}^{\prime}=k\right) \\
& =P\left(X_{n+1}=k / X_{n}=k\right)=r_{k}, \\
p_{k}^{\prime} & =1-q_{k}^{\prime}-r_{k}^{\prime} \\
& =1-q_{k}-r_{k}=p_{k}, \quad \text { for every } k \geq 1 .
\end{aligned}
$$

To express this kind of duality we will call the Markov chain $\left(X_{n}^{\prime}\right)_{n \geq 0}$, the adjoint of the Markov chain $\left(X_{n}\right)_{n \geq 0}$ and reciprocally in the case that the relation (28) is satisfied.

Equivalently for the Markov chain $\left(X_{n}^{\prime}\right)_{n \geq 0}$, the harmonic function $g^{\prime}=\left(g_{k}^{\prime}, k \geq 1\right)$ satisfies the equation

$$
\begin{gathered}
r_{0}^{\prime} \cdot g_{0}^{\prime}+q_{0}^{\prime} \cdot g_{1}^{\prime}=g_{0}^{\prime}, \\
q_{k}^{\prime} \cdot g_{k+1}^{\prime}+p_{k}^{\prime} \cdot g_{k-1}^{\prime}+r_{k}^{\prime} \cdot g_{k}^{\prime}=g_{k}^{\prime}, \quad k \geq 1 .
\end{gathered}
$$

Since $\Delta g_{k}^{\prime}=g_{k}^{\prime}-g_{k-1}^{\prime}$, for every $k \geq 1$, we have

$$
\begin{gathered}
q_{k}^{\prime} \cdot\left(\Delta g_{k+1}^{\prime}+g_{k}^{\prime}\right)+p_{k}^{\prime} \cdot g_{k}^{\prime}-p_{k}^{\prime} \cdot g_{k}^{\prime} \\
+p_{k}^{\prime} \cdot g_{k-1}^{\prime}+r_{k}^{\prime} \cdot g_{k}^{\prime}=g_{k}^{\prime}, \\
\left(p_{k}^{\prime}+q_{k}^{\prime}+r_{k}^{\prime}\right) \cdot g_{k}^{\prime}+q_{k}^{\prime} \cdot \Delta g_{k+1}^{\prime} \\
-p_{k}^{\prime} \cdot g_{k}^{\prime}+p_{k}^{\prime} \cdot g_{k-1}^{\prime}=g_{k}^{\prime}, \\
q_{k}^{\prime} \cdot \Delta g_{k+1}^{\prime}=p_{k}^{\prime} \cdot\left(g_{k}^{\prime}-g_{k-1}^{\prime}\right)=p_{k}^{\prime} \cdot \Delta g_{k}^{\prime} .
\end{gathered}
$$

If we put $\ell_{k}=\Delta g_{k+1}^{\prime} / \Delta g_{k}^{\prime}$, we get $\ell_{k}=p_{k}^{\prime} / q_{k}^{\prime}$ (with $\left.q_{k}^{\prime}=1-p_{k}^{\prime}-r_{k}^{\prime}\right), k \geq 1$, which is the equation of the definition of the sequences $\left(b_{k}\right)_{k \geq 1}$ and $\left(\gamma_{k}\right)_{k \geq 1}$ (as a multiplicative factor of the $\left.\left(b_{k}\right)_{k \geq 1}\right)$ for the Markov chain $\left(X_{n}\right)_{n \geq 0}$ such that $p_{k}^{\prime}=$ $p_{k}, r_{k}^{\prime}=r_{k}$, for every $k \geq 1$. By considering a similar approximation of that given before for the Markov chain $\left(X_{n}\right)_{n \geq 0}$, we may say that the strictly increasing harmonic functions of the Markov chain $\left(X_{n}^{\prime}\right)_{n \geq 0}$ are in correspondence with the weight representations of the Markov chain $\left(X_{n}\right)_{n \geq 0}$ such that equivalent equations of (28) are satisfied.

So we may have the following.

Proposition 5. The Markov chain $\left(X_{n}\right)_{n \geq 0}$ defined as above is transient if and only if the adjoint Markov chain $\left(X_{n}^{\prime}\right)_{n \geq 0}$ is positive recurrent and reciprocally. Moreover the adjoint Markov chains $\left(X_{n}\right)_{n \geq 0}$ and $\left(X_{n}^{\prime}\right)_{n \geq 0}$ are null recurrent simultaneously.

In particular

(i) the Markov chain $\left(X_{n}\right)_{n \geq 0}$ defined as above is transient if and only if $\left(1 / w_{0}^{\prime \prime}\right) \cdot \sum_{k=1}^{\infty} w_{k}^{\prime \prime}<+\infty$ and $\left(1 / w_{0}^{\prime \prime \prime}\right)$. $\sum_{k=1}^{\infty} w_{k}^{\prime \prime \prime}=+\infty$;

(ii) the Markov chain $\left(X_{n}^{\prime}\right)_{n \geq 0}$ defined as above is transient if and only if $\left(1 / w_{0}\right) \cdot \sum_{k=1}^{\infty} w_{k}<+\infty$ and $\left(1 / w_{0}^{\prime}\right)$. $\sum_{k=1}^{\infty} w_{k}^{\prime}<+\infty$;

(iii) the adjoint Markov chains $\left(X_{n}\right)_{n \geq 0}$ and $\left(X_{n}^{\prime}\right)_{n \geq 0}$ are null recurrent if

$$
\begin{aligned}
& \frac{1}{w_{0}} \cdot \sum_{k=1}^{\infty} w_{k}=\frac{1}{w_{0}^{\prime \prime}} \cdot \sum_{k=1}^{\infty} w_{k}^{\prime \prime}=+\infty \\
& \frac{1}{w_{0}^{\prime}} \cdot \sum_{k=1}^{\infty} w_{k}^{\prime}=\frac{1}{w_{0}^{\prime \prime \prime}} \cdot \sum_{k=1}^{\infty} w_{k}^{\prime \prime \prime}=+\infty
\end{aligned}
$$

Proof. The proof of Proposition 5 is an application mainly of Proposition 4 as well as of Proposition 3.

\section{Acknowledgment}

C. Ganatsiou is indebted to the referee for the valuable comments, which led to a significant change of the first version.

\section{References}

[1] S. Kalpazidou, Cycle Representations of Markov Processes, Springer, New York, NY, USA, 1995.

[2] J. MacQueen, "Circuit processes", Annals of Probability, vol. 9, pp. 604-610, 1981.

[3] Q. Minping and Q. Min, "Circulation for recurrent markov chains," Zeitschrift für Wahrscheinlichkeitstheorie und Verwandte Gebiete, vol. 59, no. 2, pp. 203-210, 1982.

[4] A. H. Zemanian, Infinite Electrical Networks, Cambridge University Press, Cambridge, UK, 1991.

[5] Yv. Derriennic, "Random walks with jumps in random environments (examples of cycle and weight representations)," in Probability Theory and Mathematical Statistics: Proceedings of The 7th Vilnius Conference 1998, B. Grigelionis, J. Kubilius, V. Paulauskas, V. A. Statulevicius, and H. Pragarauskas, Eds., pp. 199-212, VSP, Vilnius, Lithuania, 1999.

[6] S. Karlin and H. Taylor, A First Course in Stochastic Processes, Academic Press, New York, NY, USA, 1975. 


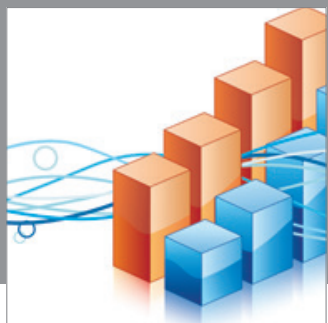

Advances in

Operations Research

mansans

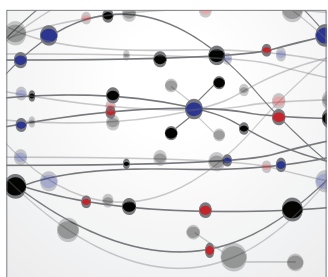

The Scientific World Journal
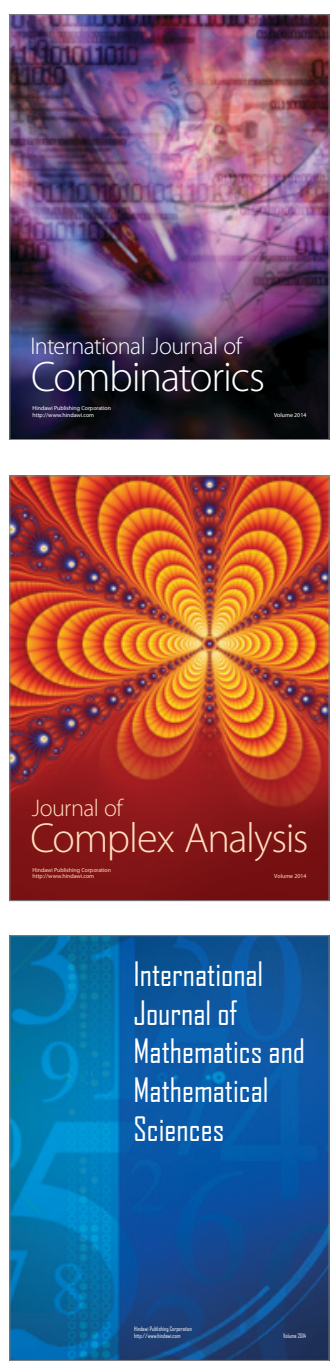
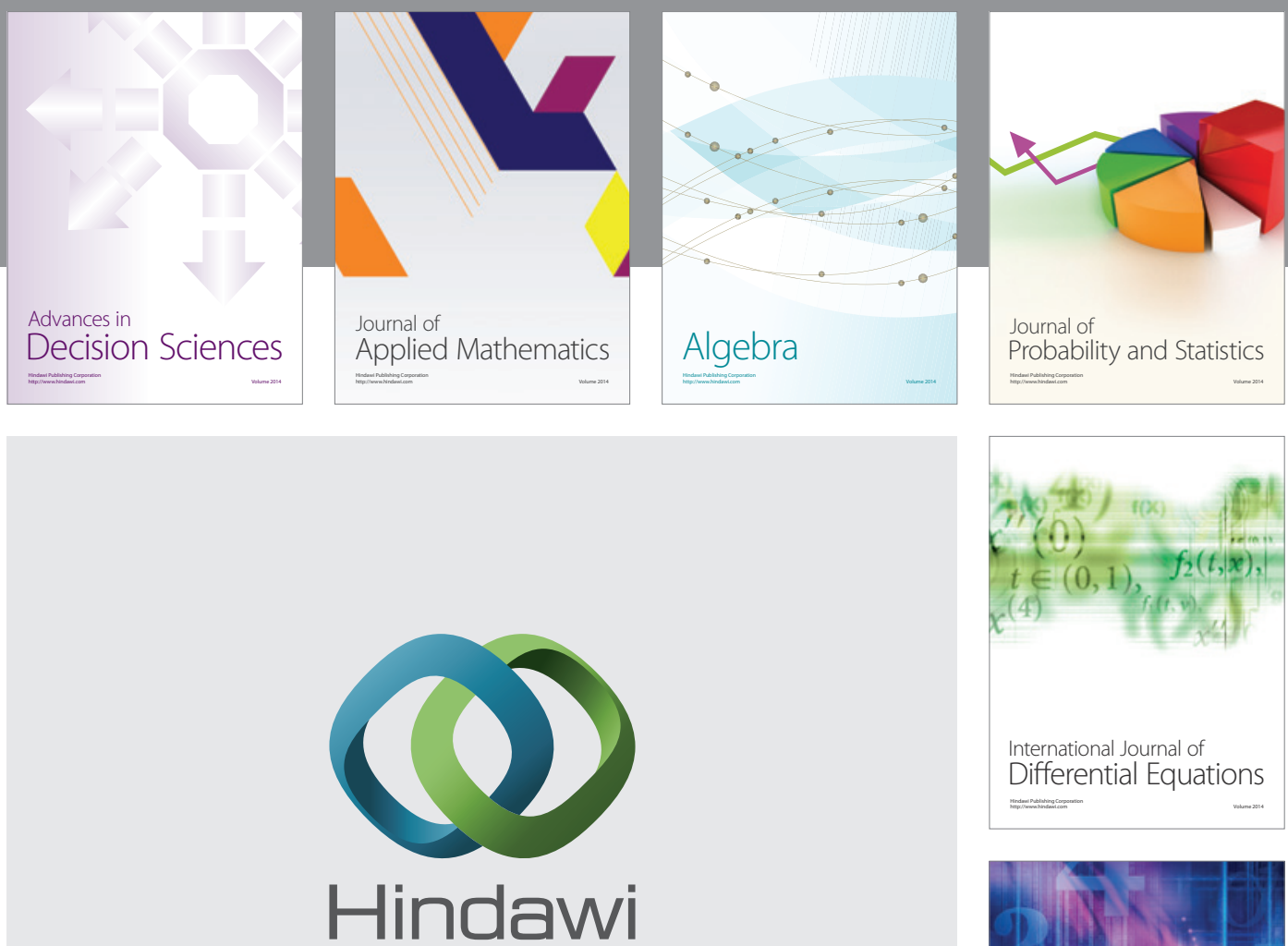

Submit your manuscripts at http://www.hindawi.com
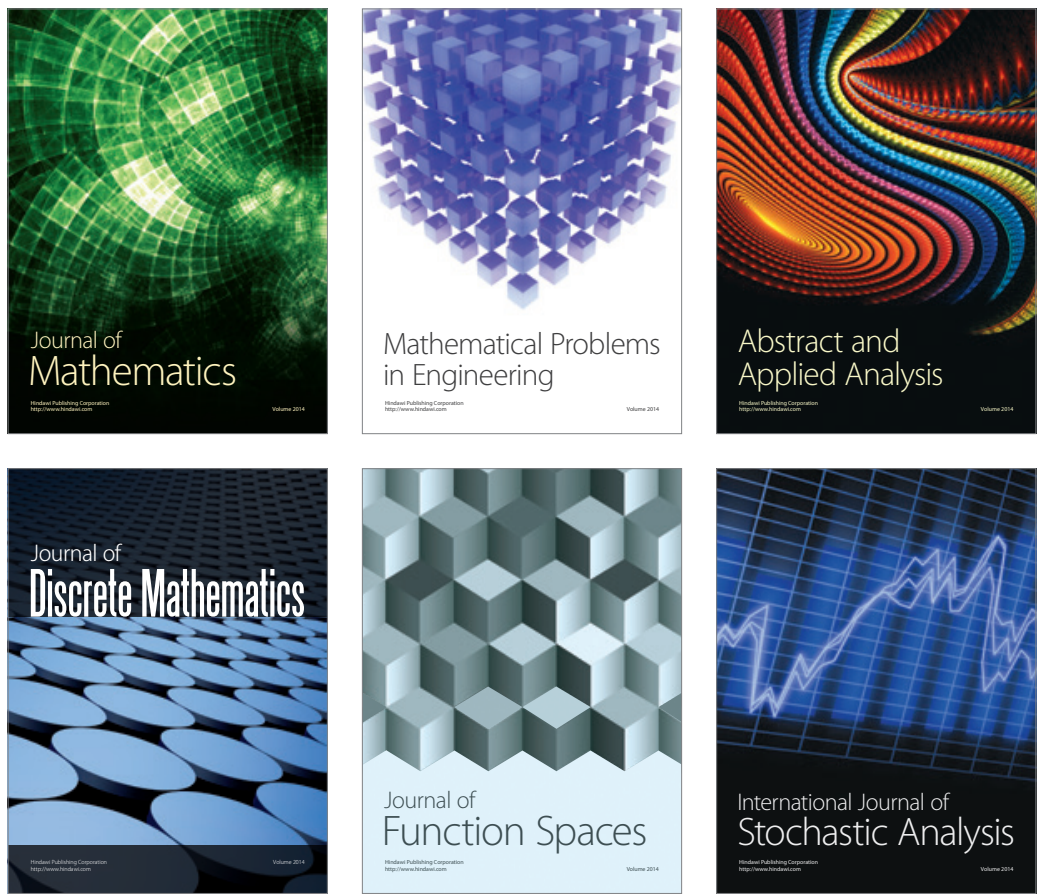

Journal of

Function Spaces

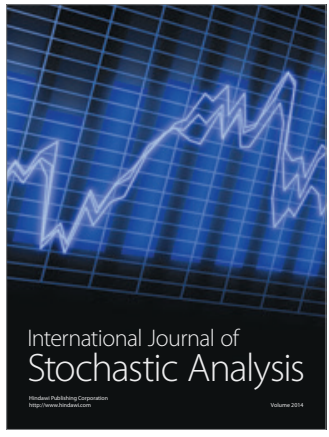

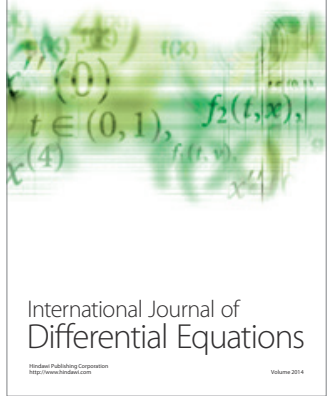
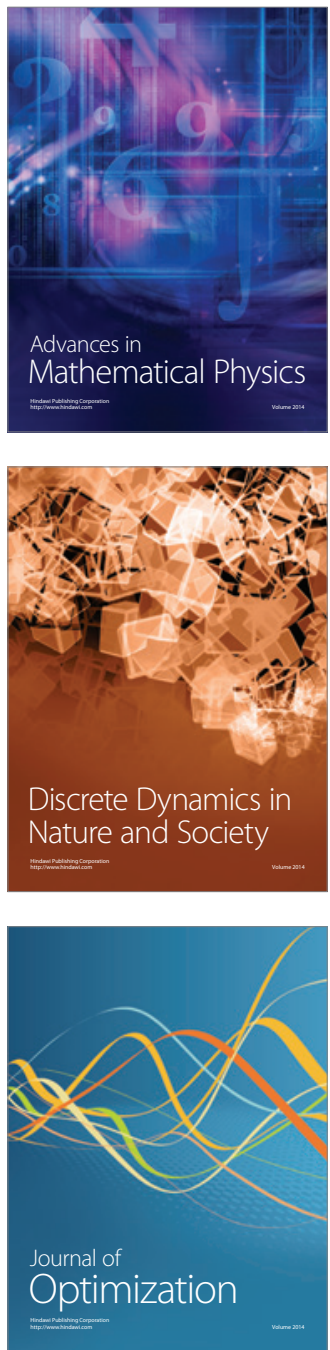